\section{Recent advances in cardiovascular research: systems biology}

\author{
Raffaella D'Alessandro, Joseph Sepe ${ }^{2}$ \\ 1 Department of Cardiothoracic and \\ Respiratory Sciences, Monaldi Hospital, \\ Naples, Italy; ${ }^{2}$ Life Sciences, University of \\ Maryland University College, Adelphi, \\ MD, USA
}

\begin{abstract}
The authors describe different bioinformatic approaches to cardiovascular research, focusing on: i) the complexity of cardiovascular diseases; ii) how does systems biology work and its application to cardiology; iii) new systems research in cardiology; iv) clinical cardiological practice; v) perspectives and limitations of systems biology in cardiology.
\end{abstract}

\section{Complexity of cardiovascular diseases}

Complex cardiovascular diseases have long been studied, but still little is known about the cellular and molecular pathogenesis underlying many conditions. In the last century, several scientific breakthroughs beginning with the Watson-Crick model of DNA have opened a window into physical and functional relationships between organelles, tying them into a unique process instead of separated compartments.

The complexity of cardiovascular function is an overall expression of its components, from myocardium to cardiomyocyte with subcellular compartments interacting for synchronized muscle contraction. For example, generating an action potential, requires the coordination of more than 20 different ion transporters and channels. ${ }^{1}$ The burden of complexity, however, is that there seems to be no limit to the number of ways this can go astray. A single error in protein synthesis or in gene expression encoding a ion channel can disrupt the entire network, as in Brugada syndrome, long QT syndrome and catecholaminergic polymorphic ventricular tachycardia. ${ }^{2}$

Today, physiological mechanisms can be studied in systems rather than single genes or proteins, using gene expression, whole genome single nucleotide polymorphism analysis, next generation re-sequencing, protein microarray, epigenetics, etc.). ${ }^{3}$

By providing a background for predictive consequences, and performing in silico simulations, computational models are establishing new ground rules for research. ${ }^{4}$

\section{How does systems biology work?}

Systems biology is based on the concept that processes can be better understood by studying networks among molecules rather than linear pathways. This prerequisite can be met by taking an interdisciplinary approach to analyze biological data.

The multi-sectorial nature of systems biology is founded upon integration of data from mathematics bioinformatics, biology and medicine.

Starting from laboratory experiments, biological information can be translated into clinical practice in a stepwise fashion, as illustrated in the following graph (Figure 1).

First, clinicians acquire information about the cardiac phenotype using animal models and patient analysis. Then researchers perform data acquisition following -omics experiments requiring statistical validation; findings can be interpreted through the consultation of scientific databases (already known associations) and ontology studies (such as the Gene Ontology database), allowing mathematical modeling of biological interactions. The final confirmation comes from animal or cellular models.

New approaches dealing with complex biological and clinical problems are based on the need to interpret -omics scale data. A number of bioinformatics tools allowing an elaboration and a visualization of results has been developed to support researchers working in that field. ${ }^{5}$

\section{Systems biology in cardiology}

The combination of data arising from clinical, cytological, tissue and organ imaging and molecular can substantiate improvements in medical sciences, such as cardiology.

The complex genetic etiology underlying cardiovascular diseases has been studied for decades, and our knowledge in this regard is rapidly increasing. In most cardiovascular diseases there is a strong interaction between genes and environmental factors, so common genetic approaches can fail to unlock molecular causes of the disease. In such phenotypes, molecular investigations performed with a systemic approach, can lead to better results, correlating the different actors playing in the specific process. ${ }^{1}$
Correspondence: Raffaella D'Alessandro, Department of Cardiothoracic and Respiratory Sciences, Monaldi Hospital, Naples, Italy.

E-mail: raffaelladalessandro@gmail.com

Key words: cardiovascular research, bioinformatic approaches.

Received for publication: 19 June 2012.

Revision received: 3 August 2012.

Accepted for publication: 17 September 2012.

This work is licensed under a Creative Commons Attribution NonCommercial 3.0 License (CC BYNC 3.0).

(C) Copyright R. D'Alessandro and J. Sepe, 2012

Licensee PAGEPress, Italy

Cardiogenetics 2012; 2:e9

doi:10.4081/cardiogenetics.2012.e9

Moreover, the development of models in cardiology is simplified by the regularity of heart function (both in spatial and in temporal terms) and by the deep knowledge of basilar myocyte biology. ${ }^{6}$

Finally, systems biology approaches can be used in molecular research to study cardiomyocyte physiology and data coming from transcriptome, genome or biochemical analysis, extending the current knowledge about physiologic mechanisms and to predict phenotypic outcome. ${ }^{7}$

\section{Applications of new systems research in cardiology}

A number of studies using the approach of systems biology in the cardiological field have been published ${ }^{1}$ an elegant example has been proposed by He et al.,$^{8}$ to integrate data about genetic, transcriptomic and proteomic studies about the origin of congenital heart diseases (CHDs).

Among the complex cardiovascular diseases, CHDs represent the most common cause of infant morbidity. Follows a description of the approach, which was based on a network analysis.

Authors used computational mining to analyze data coming from microarray experiments on CHD samples, protein-protein interactions, and genes known to be involved in CHDs to identify dysfunctional modules of proteins potentially involved in the disease. Such modules represent groups of significantly coexpressed genes.

The bioinformatic network analysis has been performed comparing proteins resistor network, ${ }^{9}$ where proteins are represented as nodes and interactions are represented as 
resistors.

The following workflow was used:

- identification of causative genes by literature studies;

- identification of target genes (i.e., differentially expressed in a significant number of patients);

- overlaying of causative genes and target genes to protein-protein interaction map to construct a network of significant interactions (dysfunctional networks);

- identification of shortest pathways connecting causative genes to target genes (different sub-networks can be found)

- assignation of information scores to genes (based on the electric model) and definition of modules (a module can contain multiple pathways and modules can be connected by pathways).

The approach allowed the authors to identify 12 modules consisting of 498 nodes and 2413 edges; each module involved a number of genes ranging from 14 to 66 genes.

Once identified the dysfunctional modules, subsequent statistical analysis correlated them with the disease phenotype, revealing the better phenotype classification accuracy of the modules than single pathways (Figure 2).

Following the preliminary analysis aimed to classify significant target genes, a functional analysis (through gene ontology classification) revealed statistically significant classes of genes identified through the computational model, showing results supporting the pathophysiologic mechanism of CHDs.

The principal module involved in CHD has provided an abundance of genes acting in anatomical structure morphogenesis, cytoskeleton organization and cellular component assembly (such as alpha-cardiac actin, dystrophin), following on the same class of several causal genes already known. The second significant module includes genes belonging to the class of signaling pathways involved in development processes (including also causal genes as NOTCH1 and JAG1).

The above mentioned modules have a central role in the resulting network, with other modules involved in heart development; conversely in the peripheral region of the network modules includes gene classes not straightly related to the pathogenesis of CHDs: this is the case of phosphorous metabolic processes, cell response to hormones, lipid metabolic processes and positive regulation of transcription, etc. Such genes are not principal actors in the development of congenital heart diseases (their role is not extremely specific), but they have a role in supporting development processes (as demonstrated by the presence of fundamental cardiac transcription factors, i.e. GATA4 and TBX5).

Possible applications of the approach are not limited to gene grouping in modules and to

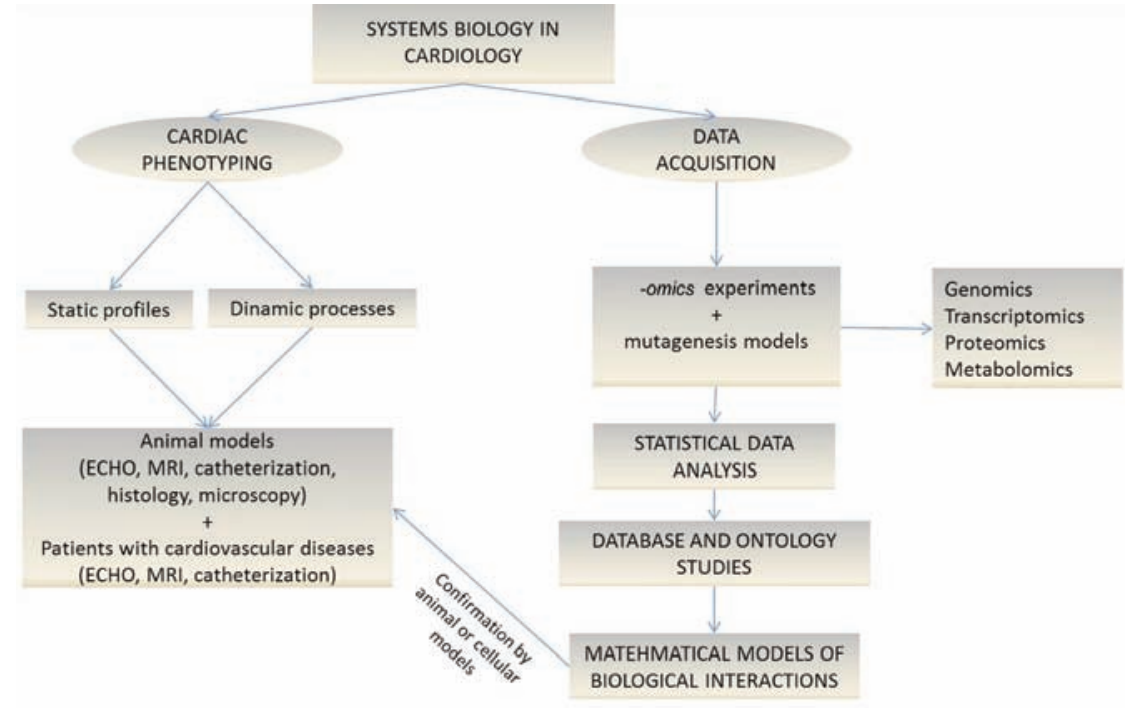

Figure 1. Correlation of clinical and experimental data to analyze a complex system.

functional classification. As discussed by authors, a wide view of the interested molecules (such as a pathway view) and some indications about other possible (not yet identified) causal genes have also been proved to provide useful insights.

The results obtained through gene ontology annotations of single target genes, have been confirmed by module-pathway crosstalk: modules previously described as central in the network have also been related to pathways regarding cardiac muscle contraction, dorsoventral axis formation, gap junctions and regulation of actin cytoskeleton.

Finally, a further application of the approach has been proposed to select potential candidate genes for the specific phenotype. The identification in the network of genes next to causal genes (in the shortest path connecting two causal genes) or being the first neighboring, with a subsequent analysis to evaluate the gene ontology similarity to the causal gene in the same module has resulted in 60 candidate genes. ${ }^{8}$

\section{Implications for clinical cardio- logical practice}

Developments in cardiologic molecular research, as in other fields, are aimed to reach the final goal of improving current clinical practice.

However, complex biological networks are currently in an early stage of clinical application.

The most important objective in the years to come is to apply this to personalized cardiolo-

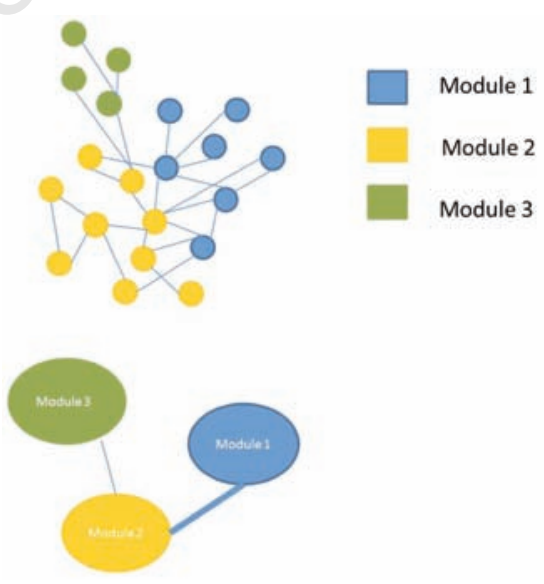

Figure 2. Example of networks and modules.

gy, with predictive and individualized clinical assistance followed by a more specific diagnosis and therapy; such a new concept of medicine can be obtained through the use, the interpretation and the connection of data coming from high throughput experiments (the previously defined -omics), which allow the analysis of the entire scenario in which a disease arises. ${ }^{10}$

The increasing body of information about multi-factorial molecular basis of diseases involves the necessity of considering the entire system simultaneously, leaving the reductionist approach based on the identification of a single (or few) molecule responsible for a specific. ${ }^{11}$

In the field of clinical cardiology, developments on the basis of a better understanding 
of the molecular background of a subject, could lead to a detailed prediction of biomarkers involved in heart diseases: metabolite profiles can provide details about physiology and specific conditions promoting the disease and correlate therapy response to metabolite levels. An already applied (extremely limited) example of metabolomics application in clinical practice is the predictive value of cholesterol levels. $^{12}$

\section{Perspectives and limitations of systems biology in cardiology}

Nowadays, systems biology is a growing field of research and studies using this approach exploit recent advances in computational analysis applied to biological phenomena. As models describing processes in other scientific areas, also the approach to cardiology is characterized by bias due to the stiffness of a computational model in comparison to real, living organs. However, systems biology has limitations arising from the need to analyze high throughput data using appropriate statistical models to evaluate the correctness of the results obtained and the necessity of designing confirmation model experiments. ${ }^{7}$

Failing models, however, may not necessarily be detrimental because they spark direct research into the missing pieces of the puzzle and became a tool for testing the our comprehension of biological processes. ${ }^{6}$

The system perspective can be applied to clinical and diagnostic reality, as standard approaches (investigating only a gene/protein or a set of genes/proteins) can lose information about genomic/proteomic interactions. So, not only clinical diagnosis can become more specific with a more detailed classification of molecular medicine (also with overlapping phenotypes, e.g. Noonan and LEOPARD syndromes), but also the goal of an individualized medicine, which is currently far off, but can be undoubtedly better be approached with the implementation of systems researches. ${ }^{13,14}$

\section{References}

1. Lusis AJ, Weiss JN. Cardiovascular networks: systems-based approaches to cardiovascular disease. Circulation 2010;121: 157-70.

2. Martin CA, Matthews GD, Huang CL. Sudden cardiac death and inherited channelopathy: the basic electrophysiology of the myocyte and myocardium in ion channel disease. Heart 2012;98:536-43.

3. Dewey FE, Wheeler MT, Ashley EA. Systems biology of heart failure, challenges and hopes. Curr Opin Cardiol 2011;26:314-21.

4. Louridas GE, Kanonidis IE, Lourida KG. Systems biology in heart diseases. Hippokratia 2010;14:10-6.

5. Gehlenborg N, O'Donoghue SI, Baliga NS, et al. Visualization of omics data for systems biology. Nat Methods 2010;7 Suppl 3:S56-68.
6. Quinn TA, Kohl P. Systems biology of the heart: hype or hope? Ann N Y Acad Sci 2011;1245:40-3.

7. Sperling SR. Systems biology approaches to heart development and congenital heart disease. Cardiovasc Res 2011;91:269-78.

8. He D, Liu ZP, Chen L. Identification of dysfunctional modules and disease genes in congenital heart disease by a networkbased approach. BMC Genomics 2011;12:592.

9. Missiuro PV, Liu K, Zou L, et al. Information flow analysis of interactome networks. PLoS Comput Biol 2009;5:e 1000350.

10. Louridas GE, Lourida KG. The new biology: a bridge to clinical cardiology. Hippokratia 2012;16:106-12.

11. Molina F, Dehmer M, Perco P, et al. Systems biology: opening new avenues in clinical research. Nephrol Dial Transplant 2010;25:1015-8.

12. Ginsburg GS, Donahue MP, Newby LK. Prospects for personalized cardiovascular medicine: the impact of genomics. J Am Coll Cardiol 2005;46:1615-27.

13. Itadani H, Mizuarai S, Kotani H. Can systems biology understand pathway activation? Gene expression signatures as surrogate markers for understanding the complexity of pathway activation. Curr Genomics 2008;9:349-60.

14. Loscalzo J, Kohane I, Barabasi AL. Human disease classification in the postgenomic era: a complex systems approach to human pathobiology. Mol Syst Biol 2007;3:124. 